\title{
Minimally Invasive Surgery versus Open Surgery for Adolescent Idiopathic Scoliosis: A Systematic Review and Meta-Analysis
}

\author{
Deepak Neradi*, Vishal Kumar*, Sunil Kumar, Praveen Sodavarapu, Vijay Goni, Sarvdeep Singh Dhatt \\ Department of Orthopaedics, Postgraduate Institute of Medical Education and Research, Chandigarh, India
}

\begin{abstract}
Minimally invasive surgery (MIS) is being recommended over more invasive methods. MIS advantages are less time in the operating room, less blood loss, a shorter recovery time, and shorter length of stay. A systematic review and meta-analysis were performed using the literature from minimally invasive and open surgery for adolescent idiopathic scoliosis (AIS). We conducted this analysis to see whether MIS has advantages over traditional surgery. A systematic review was conducted using PubMed, Embase, and Scopus to find articles comparing minimally invasive and open surgery techniques for AIS patients. Data extraction and meta-analysis were completed. The primary data points collected were correction rate and functional outcomes, including perioperative and postoperative parameters. A total of six studies were included in the final analysis. The MIS group had 123 patients, and the open surgery group had 150 patients. The correction rate and functional outcomes favored the open surgery group with a mean difference of $4.60195 \%$ confidence interval [Cl], 0.08 to 9.12 ) and $0.11(95 \% \mathrm{Cl}, 0.04$ to 0.17$)$, respectively. The duration of surgery, blood loss, number of patients requiring transfusion, and analgesic requirements favored the MIS group with a significant difference. Open surgery is better than MIS in achieving a better correction rate and good functional outcomes. MIS is better over open surgery when perioperative parameters are considered.
\end{abstract}

Keywords: Minimally invasive surgery; Open surgery; Adolescent idiopathic scoliosis; Outcomes; Meta-analysis

\section{Introduction}

Scoliosis is a condition where the spine is laterally bent from the central axis. The three types of scoliosis are infantile, juvenile, and adolescent. Adolescent scoliosis is the most common and more common in females. Various factors have been implicated in its cause, such as calmodulin [1], transforming growth factor beta [2], estrogen [3], melatonin, and elastin fiber abnormalities. An idiopathic scoliosis diagnosis comprises $90 \%$ of patients with adoles- cent scoliosis [4]. The deformity grows rapidly as the child grows, and then it stops or progresses at a slower rate. Stokes et al. [5] suggested that curve progression results from a vicious cycle of asymmetrical loading. These patients have a complex three-dimensional (3D) deformity in all three planes, including the kyphosis-sagittal plane, rotation-axial plane, and scoliosis-coronal plane. These deformities result in cosmetic disturbance, decreased pulmonary functions, and short stature. Most of the time, adolescent idiopathic scoliosis (AIS) can be conservatively

Received Nov 29, 2020; Revised Jan 25, 2021; Accepted Jan 27, 2021

Corresponding author: Sarvdeep Singh Dhatt

Department of Orthopaedics, Postgraduate Institute of Medical Education and Research, Chandigarh, 160012, India

Tel: +91-9815126600, Fax: +91-172-2744401, E-mail: dhatt.sarvdeeps@pgimer.edu.in

*These two authors contributed equally to this work. 
managed. Surgery is done for cosmetic reasons and if patients have debilitating symptoms. Traditional surgery involves a posterior approach, opening vertebra at all deformed levels, and using pedicle screws to correct the deformity. This approach is the gold standard resulting in correction of the deformity. All surgery, including open scoliosis surgery, is associated with complications. Traditional open scoliosis surgery complications include dural tears, neural injury, increased operating time, blood loss, increased duration of hospital stay, and position-related complications like brachial plexus injury and visual loss [6-8].

Surgeons recommend a minimally invasive technique for their patients based on lower infection rates, a shorter hospital stay, and an earlier return to normal activities [9]. Studies also show that minimally invasive surgery (MIS) is associated with fewer complications [10]. The evidence gathered to date is not enough to suggest that MIS is superior to traditional open surgery. There are very few comparative studies comparing MIS to open surgery for AIS. We did find one systematic review and meta-analysis comparing MIS and open surgery for AIS patients [11]. The meta-analysis included MIS patients with patients operated on using video-assisted thoracoscopic surgery (VATS) in the same group, which may have lowered the study's quality. We were also unable to find any randomized controlled trials (RCT) on the same topic. We worked to find evidence by conducting a systematic review and meta-analysis comparing MIS and open surgery for AIS patients.

\section{Methodology}

A comprehensive review according to the Preferred Reporting Items for Systematic Reviews and MetaAnalyses (PRISMA) guidelines for the bibliographic database PubMed was performed [12]. We also followed the Cochrane guidelines at every step while conducting this meta-analysis. The current study is registered with PROSPERO reg.no: CRD42020217823. Databases like PubMed, Embase, and Scopus were used to search for relevant articles comparing MIS and open surgery for AIS. Two reviewers (D.N. and P.S.) searched the databases for related articles from inception to 22nd October 2020. Additionally, all PubMed citations and bibliography were also checked for similar articles.

\section{Inclusion and exclusion criteria}

We selected studies that satisfied the following inclusion criteria: (1) comparative studies (RCT, non-randomized clinical trials, case-controlled studies, cohort studies, and other comparative studies) where MIS had been compared with standard posterior open surgery, (2) studies that dealt with surgery for AIS, and (3) English language articles. Excluded studies were (1) dealing with adult scoliosis surgery, (2) studies dealing with MIS using VATS surgery, (3) studies comparing anterior and posterior surgery, (4) studies dealing with conservative or operative treatment, (5) non-comparative studies like case series, and (6) non-English language articles.

\section{Study selection}

First, two independent reviewers (D.N. and V.K.) screened the study titles, abstract, and other publication details like author, year of publication, journal name, and more for duplication. After that, the titles and abstracts of the remaining studies were checked for eligibility based on our inclusion and exclusion criteria. The full texts of potentially eligible studies were then retrieved and independently accessed for eligibility by the two reviewers. Any disagreements between them over the eligibility of a particular study were resolved through mutual discussion and, when unresolved, by discussing with a third reviewer (S.S.D.).

\section{Data extraction}

Two independent authors (D.N. and V.K.) extracted the following information from all the articles: lead author, publication year, country of study, type of study, number of patients enrolled and followed, follow-up duration, mean age of participants, male/female ratio, diagnosis, intraoperative parameters (duration of surgery, blood loss, and transfusion requirement), correction rate, postoperative clinical function, and incidence of complications (implant failure, infection, and nerve injuries). All the data were extracted to a standardized excel sheet.

\section{Quality assessment}

All the studies were categorized by their level of evidence. The methodological quality of RCTs was done by using the Cochrane risk of bias assessment tool and non- 
randomized studies (case-control and cohort studies) by the methodological index for non-randomized studies (MINORS) [13]. Two independent authors (D.N. and P.S.) completed this work, and any disagreements between them were resolved through mutual discussion.

\section{Statistical analysis}

Meta-analysis was performed using Review Manager ver. 5.4 software (Cochrane Collaboration, London, UK). The standard mean difference or weighted mean difference with $95 \%$ confidence intervals (CIs) was calculated for continuous variables. An odds ratio (OR) and risk ratio with $95 \%$ CIs were used for dichotomous variables. The level of significance was set to a $p$-value $<0.05$. Whether to use a fixed-effects or random-effects model was decided based on heterogeneity, evaluated by $\chi^{2}$ test and $I^{2}$ statistics. If the statistical heterogeneity was significant ( $p$-value $<0.10$ or $I^{2}>50 \%$ ), the random-effects model was used; otherwise, the fixed-effects model was used.

\section{Results}

\section{Literature search}

A total of 481 records were identified in the initial literature search, of which 81 were duplications. Then, out of the remaining 400, 393 were excluded basaed on title and abstract following the exclusion criteria. Seven fulltext articles were identified as eligible. We were unable to access the full text of one article. Finally, six studies (one prospective and five retrospective comparative studies) met our inclusion criteria and were included for systematic review and meta-analysis [14-19] (Fig. 1).

\section{Risk assessment}

As mentioned in the methodology, risk assessment was done using MINORS tool. The results are summarized in Table 1.

\section{Study characteristics}

The studies included in our analysis were published between 2010 and 2020. Two of these studies were from China and one from Spain, Canada, Poland, and New York. There were five retrospective studies and one pro-

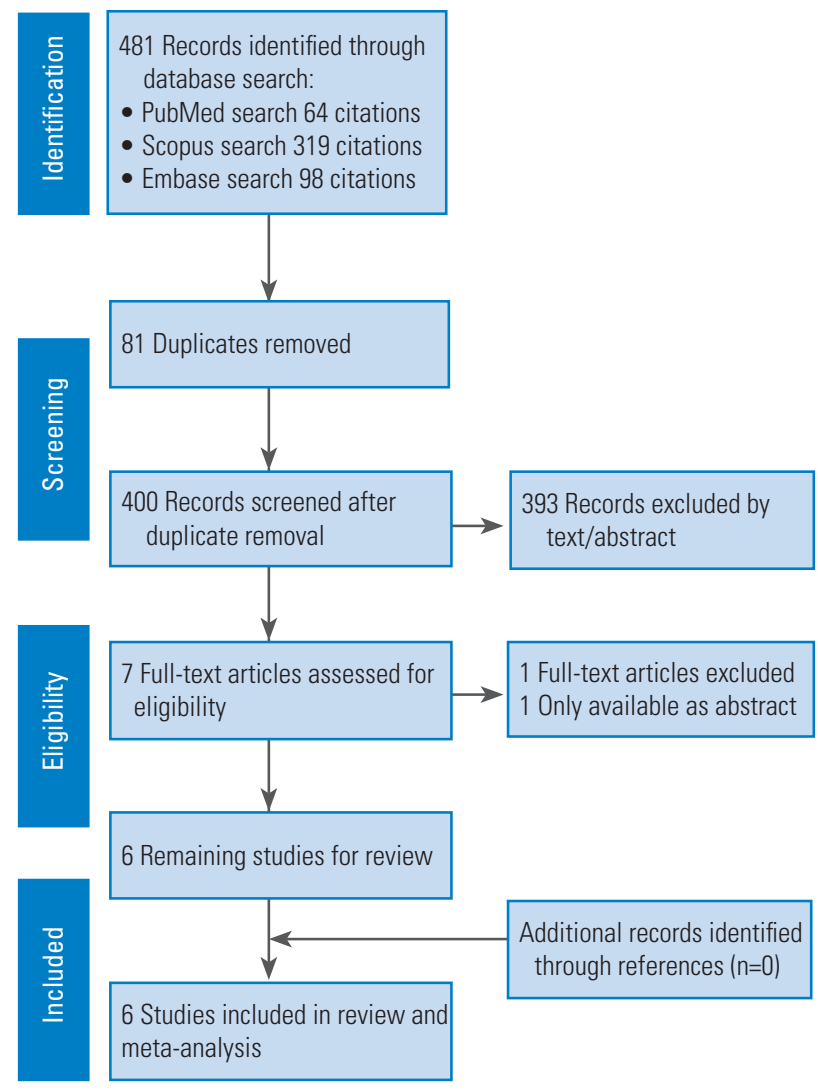

Fig. 1. Showing PRISMA (Preferred Reporting Items for Systematic Reviews and Meta-Analyses) flowchart.

spective study. The major characteristics of the studies are mentioned in Table 2. A total of 253 patients (MIS: 123, open surgery: 130) were enrolled and evaluated. In all studies, the MIS techniques used were similar, with multiple posterior midline skin incisions. In all studies, the flexibility of the curve was $>50 \%$. All studies mentioned no significant difference between the two groups when considering sagittal and coronal balance. While the types of curves were different in each study, there was no significant difference between the two groups when comparing the curves. The majority of studies included were retrospective; there were no patients with lost followup information. The minimum follow-up period of the included studies was 2 years. Data regarding radiological parameters at the final follow-up are presented in Table 3 , and perioperative data and complications are presented in Table 4 .

The meta-analysis of primary outcomes included correction rate and functional outcomes, shown in Fig. 2. The meta-analysis of perioperative data included surgery duration, blood loss, number of patients requiring blood 
Table 1. Risk of bias assessment using methodological items for non-randomized studies

\begin{tabular}{|c|c|c|c|c|c|c|}
\hline Items & $\begin{array}{l}\text { Gómez et al. } \\
\text { [16] (2013) }\end{array}$ & $\begin{array}{l}\text { Miyanji et al. } \\
\text { [14] (2015) }\end{array}$ & $\begin{array}{l}\text { Sarwahi et al. } \\
\text { [15] (2014) }\end{array}$ & $\begin{array}{l}\text { Si et al. [18] } \\
(2020)\end{array}$ & $\begin{array}{l}\text { Urbanski et al. } \\
\text { [17] (2019) }\end{array}$ & $\begin{array}{l}\text { Zhu et al. } \\
\text { [19] (2017) }\end{array}$ \\
\hline 1. Clearly stated aim & 2 & 2 & 2 & 2 & 2 & 2 \\
\hline 2. Inclusion of consecutive patients & 2 & 2 & 2 & 2 & 2 & 2 \\
\hline 3. Prospective collection of data & 1 & 0 & 0 & 0 & 0 & 0 \\
\hline 4. Endpoints appropriate to the aim of the study & 2 & 2 & 2 & 2 & 2 & 2 \\
\hline 5. Unbiased assessment of the study endpoint & 2 & 2 & 2 & 2 & 2 & 2 \\
\hline 6. Follow-up period appropriate to the aim of the study & 1 & 1 & 1 & 1 & 1 & 1 \\
\hline 7. Loss to follow-up less than $5 \%$ & 1 & 1 & 1 & 1 & 1 & 1 \\
\hline 8. Prospective calculation of sample size & 1 & 0 & 0 & 0 & 0 & 0 \\
\hline 9. An adequate control group & 1 & 1 & 1 & 1 & 1 & 1 \\
\hline 10. Contemporary groups & 2 & 2 & 2 & 2 & 2 & 2 \\
\hline 11. Baseline equivalence of groups & 1 & 2 & 2 & 2 & 2 & 2 \\
\hline 12. Adequate statistical analysis & 2 & 2 & 2 & 2 & 2 & 2 \\
\hline Total & 18 & 17 & 17 & 17 & 17 & 17 \\
\hline Risk & $L R$ & LR & $L R$ & $L R$ & LR & LR \\
\hline
\end{tabular}

LR, low risk.

Table 2. Showing basaline characters of included studies

\begin{tabular}{|c|c|c|c|c|c|c|}
\hline Author & Journal & Country & $\begin{array}{l}\text { Type of } \\
\text { article }\end{array}$ & Sample size & Age (yr) & Sex (M:F) \\
\hline Gómez et al. [16] (2013) & Coluna/Columna & Spain & Prospective & MIS (10), open (10) & MIS (14.3 [12.1-16.8]), open (15.3 [11-17.4]) & - \\
\hline Miyanji et al. [14] (2015) & Semin Spine Surg & Canada & Retrospective & MIS (23), open (23) & MIS (16.8 [14-20]), open (16.4 [13-19]) & MIS (3:20), open (4:19) \\
\hline Sarwahi et al. [15] (2014) & Clin Spine Surg & USA & Retrospective & MIS (7), open (15) & MIS (14.31), open (15.26) & MIS (1:6), open (2:13) \\
\hline Si et al. [18] (2020) & Eur Spine J & China & Retrospective & MIS (64), open (48) & 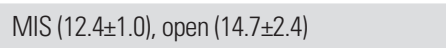 & MIS (20:44), open (14:34) \\
\hline Urbanski et al. [17] (2019) & $\begin{array}{l}\text { Arch Orthop Trauma } \\
\text { Surg }\end{array}$ & Poland & Retrospetive & MIS (4), open (4) & MIS (15.5 \pm 2.06$)$, open $(21.25 \pm 9.98)$ & MIS (4:0), open (3:1) \\
\hline Zhu et al. [19] (2017) & J Neurosurg Pediatr & China & Retrospective & MIS (15), open (30) & 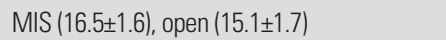 & MIS (2:13), open (3:27) \\
\hline
\end{tabular}

Values are presented as number, median (range), or mean \pm standard deviation or number (\%), unless otherwise stated.

$\mathrm{M}$, male; F, female; MIS, minimally invasive surgery.

transfusion, and number of levels fused shown in Fig. 3. The meta-analysis of postoperative parameters included length of stay, number of days patients required analgesics, and complications shown in Fig. 4.

\section{Correction rate}

All six included studies provided data regarding the correction rate at the final follow-up. There were 123 patients in the MIS group and 130 patients in the open group. The forest plot in Fig. 1A favored the open surgery group with respect to the correction rate at the final follow-up, but the difference was not significant ( $p=0.08$; mean difference,
3.86; $95 \% \mathrm{CI}, 0.45$ to 8.18$)$. As the heterogeneity was more than $50 \%$, we used the random-effects model. Out of the six included studies, Si et al. [18] had the largest sample size and could influence the results as a whole, sensitivity analysis by not including this study was completed, and results are shown in Fig. 1B. The results show that the correction rate is significantly different, favoring the open surgery group ( $p=0.05$; mean difference, $4.60 ; 95 \%$ CI, 0.08 to 9.12 ).

\section{Scoliosis Research Society-22 score at final follow-up}

Three out of six studies mentioned Scoliosis Research 
Table 3. Showing radiological parameters of included studies

\begin{tabular}{|c|c|c|c|c|c|c|c|c|}
\hline \multirow{2}{*}{ Author } & \multirow{2}{*}{ Category } & \multicolumn{2}{|c|}{ Cobbs $\left({ }^{\circ}\right)$} & \multirow{2}{*}{$\begin{array}{c}\text { Correction } \\
\text { rate }(\%)\end{array}$} & \multicolumn{2}{|c|}{ Kyphosis $\left({ }^{\circ}\right)$} & \multicolumn{2}{|c|}{ Sagittal balance $\left({ }^{\circ}\right)$} \\
\hline & & Preop & Postop & & Preop & Postop & Preop & Postop \\
\hline \multirow[t]{2}{*}{ Gómez et al. [16] (2013) } & MIS (n=10) & $60 \pm 4.5$ & - & $81 \pm 4.0$ & $25 \pm 7.25$ & $17 \pm 2.5$ & - & - \\
\hline & Open $(n=10)$ & $56 \pm 5.25$ & - & $80 \pm 7.0$ & $20 \pm 5.0$ & $22 \pm 2.25$ & - & - \\
\hline \multirow[t]{2}{*}{ Miyanji et al. [14] (2015) } & MIS (n=23) & $56.7 \pm 1.62$ & $23.9 \pm 1.68$ & $58.1 \pm 2.41$ & $20.5 \pm 2.08$ & $22.9 \pm 1.9$ & NA & NA \\
\hline & Open (n=23) & $58.1 \pm 1.57$ & $18.7 \pm 1.02$ & $68.0 \pm 1.45$ & $22.6 \pm 3.38$ & $21.0 \pm 1.32$ & NA & NA \\
\hline \multirow[t]{2}{*}{ Sarwahi et al. [15] (2014) } & MIS & $48.00 \pm 2.25$ & $10.00 \pm 1.5$ & $79.25 \pm 2.78$ & $22.00 \pm 3.00$ & $24.00 \pm 1.62$ & $5.98 \pm 0.21$ & $3.83 \pm 0.42$ \\
\hline & Open & $46.00 \pm 1.0$ & $7.00 \pm 1.0$ & $84.78 \pm 2.22$ & $24.00 \pm 3.00$ & $21.00 \pm 1.05$ & $4.39 \pm 0.66$ & $2.72 \pm 1.42$ \\
\hline \multirow[t]{2}{*}{ Si et al. [18] (2020) } & MIS & $50.7 \pm 8.8$ & $17.4 \pm 8.5$ & $65.0 \pm 17.6$ & $29.2 \pm 9.4$ & $17.50 \pm 8.80$ & - & - \\
\hline & Open & $48.0 \pm 8.4$ & $17.2 \pm 10.4$ & $64.4 \pm 19.7$ & $28.7 \pm 7.1$ & $17.8 \pm 8.2$ & - & - \\
\hline \multirow[t]{2}{*}{ Urbanski et al. [17] (2019) } & $\operatorname{MIS}(n=4)$ & $57.25 \pm 10.64$ & NA & $68.25 \pm 6.8$ & $23.6 \pm 7.61$ & $26.07 \pm 8.53$ & $22.1 \pm 9.94$ & $22.925 \pm 18.8$ \\
\hline & Open $(n=4)$ & $47 \pm 7.78$ & NA & $78 \pm 8.8$ & $37 \pm 16.06$ & $32.40 \pm 12.51$ & $53.5 \pm 39.78$ & $30.22 \pm 14.47$ \\
\hline \multirow[t]{2}{*}{ Zhu et al. [19] (2017) } & MIS & $48.3 \pm 4.2$ & $11.1 \pm 4.3$ & $77.1 \pm 8.9$ & $20.2 \pm 6.1$ & $25.2 \pm 6.2$ & $-31.0 \pm 24.2$ & $-36.3 \pm 20.7$ \\
\hline & Open & $50.9 \pm 5.4$ & $12.0 \pm 3.1$ & $76.5 \pm 7.0$ & $16.5 \pm 6.8$ & $22.9 \pm 7.5$ & $-12.0 \pm 6.2$ & $-15.4 \pm 12.1$ \\
\hline
\end{tabular}

Values are presented as mean \pm standard deviation.

Preop, preoperative; Postop, postoperative; MIS, minimally invasive surgery; NA, not available.

Table 4. Showing perioperative data and complication

\begin{tabular}{|c|c|c|c|c|c|c|c|}
\hline Author & Category & $\begin{array}{l}\text { No. of levels } \\
\text { fused }\end{array}$ & $\begin{array}{c}\text { Duration of } \\
\text { surgery (min) }\end{array}$ & Blood loss (mL) & $\begin{array}{l}\text { Length of } \\
\text { stay (day) }\end{array}$ & $\begin{array}{l}\text { PCA/opioid } \\
\text { intake }\end{array}$ & Complications \\
\hline \multirow[t]{2}{*}{ Gómez et al. [16] (2013) } & MIS & NA & $366 \pm 168$ & $270 \pm 95$ & $7.6 \pm 1.0$ & NA & NA \\
\hline & Open & NA & $204 \pm 108$ & $720 \pm 177.5$ & $7.1 \pm 1.25$ & NA & NA \\
\hline \multirow[t]{2}{*}{ Miyanji et al. [14] (2015) } & MIS & 10.2 & $475.3 \pm 13.25$ & $261.5 \pm 20.89$ & $4.4 \pm 0.15$ & $3.4 \pm 0.19$ & 5 \\
\hline & Open & 12.2 & $346.4 \pm 15.64$ & $471.7 \pm 36.09$ & $5.9 \pm 0.20$ & $3.7 \pm 0.21$ & 1 \\
\hline \multirow[t]{2}{*}{ Sarwahi et al. [15] (2014) } & MIS & NA & $538.8 \pm 22.2$ & $600.00 \pm 137.5$ & $8.00 \pm 0.75$ & $6.00 \pm 1.0$ & 6 \\
\hline & Open & NA & $424.2 \pm 144$ & $800.00 \pm 162.5$ & $7.00 \pm 0.5$ & $6.00 \pm 0.5$ & 13 \\
\hline \multirow[t]{2}{*}{ Si et al. [18] (2020) } & MIS & $8.4 \pm 2.3$ & $361 \pm 95$ & $502 \pm 218$ & NA & NA & 10 \\
\hline & Open & $6.2 \pm 2.6$ & $275 \pm 43$ & $808 \pm 520$ & NA & NA & 8 \\
\hline \multirow[t]{2}{*}{ Urbanski et al. [17] (2019) } & MIS & $6.5 \pm 0.86$ & $285 \pm 47.56$ & $138.75 \pm 50.04$ & $3.75 \pm 0.43$ & $2 \pm 0.7$ & NA \\
\hline & Open & $5.75 \pm 0.43$ & $242 \pm 44.51$ & $450 \pm 106.06$ & $7 \pm 3$ & $3.25 \pm 0.43$ & NA \\
\hline \multirow[t]{2}{*}{ Zhu et al. [19] (2017) } & MIS & $4.9 \pm 0.5$ & $252 \pm 96$ & $153 \pm 97$ & NA & NA & 2 \\
\hline & Open & $5.7 \pm 0.5$ & $192 \pm 30$ & $418 \pm 126$ & NA & NA & 0 \\
\hline
\end{tabular}

Values are presented as mean \pm standard deviation.

PCA, patient-controlled analgesia; MIS, minimally invasive surgery; NA, not available.

Society-22 (SRS-22) scoring at the final follow-up for 102 patients in the MIS group and 101 patients in the open surgery group. We used functional score values in the SRS-22 score for quantitative analysis, and the results are shown in Fig. 1C. Since the heterogeneity was low, we used the fixed-effects model, and the meta-analysis favored the open group ( $p<0.001$; mean difference, 0.11 ; $95 \%$ CI, 0.04 to 0.17$)$.

\section{Duration of surgery}

All six included studies mentioned surgical duration, 123 patients in the MIS group, and 130 patients in the open surgery group. As there was high heterogeneity, we used a random-effects model. The quantitative analysis results favoring the open surgery group are shown in Fig. 3A ( $p$-value $<0.00001$; mean difference, $95.06 ; 95 \%$ CI, 61.43 to 128.70 ). 
(A)

\begin{tabular}{|c|c|c|c|c|c|c|c|c|c|c|}
\hline \multirow{2}{*}{ Study } & \multicolumn{2}{|c|}{ MIS } & \multicolumn{2}{|c|}{ Open } & \multirow{2}{*}{$\begin{array}{c}\text { Weight } \\
(\%)\end{array}$} & \multirow{2}{*}{$\begin{array}{c}\text { Mean difference } \\
\text { IV, random, } 95 \% \mathrm{Cl}\end{array}$} & \multirow{2}{*}{\multicolumn{4}{|c|}{$\begin{array}{c}\text { Mean difference } \\
\mathrm{IV} \text {, random, } 95 \% \mathrm{Cl}\end{array}$}} \\
\hline & Mean $\pm S D$ & Total & Mean \pm SD & Total & & & & & & \\
\hline Gómez et al. [16] (2013) & $81 \pm 4$ & 10 & $80 \pm 7$ & 10 & 17.1 & $1.00(-4.00$ to 6.00$)$ & & - & & \\
\hline Miyanji et al. [14] (2015) & $58.1 \pm 2.41$ & 23 & $68 \pm 1.45$ & 23 & 21.9 & $-9.90(-11.05$ to -8.75$)$ & & $=$ & & \\
\hline Sarwahi et al. [15] (2014) & $79.25 \pm 2.78$ & 7 & $84.78 \pm 2.22$ & 15 & 20.9 & $-5.53(-7.88$ to -3.18$)$ & & - & & \\
\hline Si et al. [18] (2020) & $65 \pm 17.6$ & 64 & $64.4 \pm 19.7$ & 48 & 14.0 & $0.60(-6.45$ to 7.65$)$ & & & & \\
\hline Urbanski et al. [17] (2019) & $68.25 \pm 6.8$ & 4 & $78 \pm 8.8$ & 4 & 9.2 & $-9.75(-20.65$ to 1.15$)$ & & 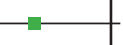 & & \\
\hline Zhu et al. [19] (2017) & $77.1 \pm 8.9$ & 15 & $76.5 \pm 7$ & 30 & 16.9 & $0.60(-4.55$ to 5.75$)$ & & - & & \\
\hline Total $(95 \% \mathrm{Cl})$ & & 123 & & 130 & 100.0 & $-3.86(-8.18$ to 0.45$)$ & & & & \\
\hline $\begin{array}{l}\text { Heterogeneity: } \text { Ta }^{2}=21.80 ; \chi^{2}=4 \\
\text { Test for overall effect: } Z=1.75 \text { (p }\end{array}$ & $=5(p<0.0$ & ${ }^{2}=8$ & & & & & -20 & $\begin{array}{l}-10 \\
\text { Open }\end{array}$ & $\begin{array}{r}1 \\
10 \\
\text { MIS }\end{array}$ & 20 \\
\hline
\end{tabular}

(B)

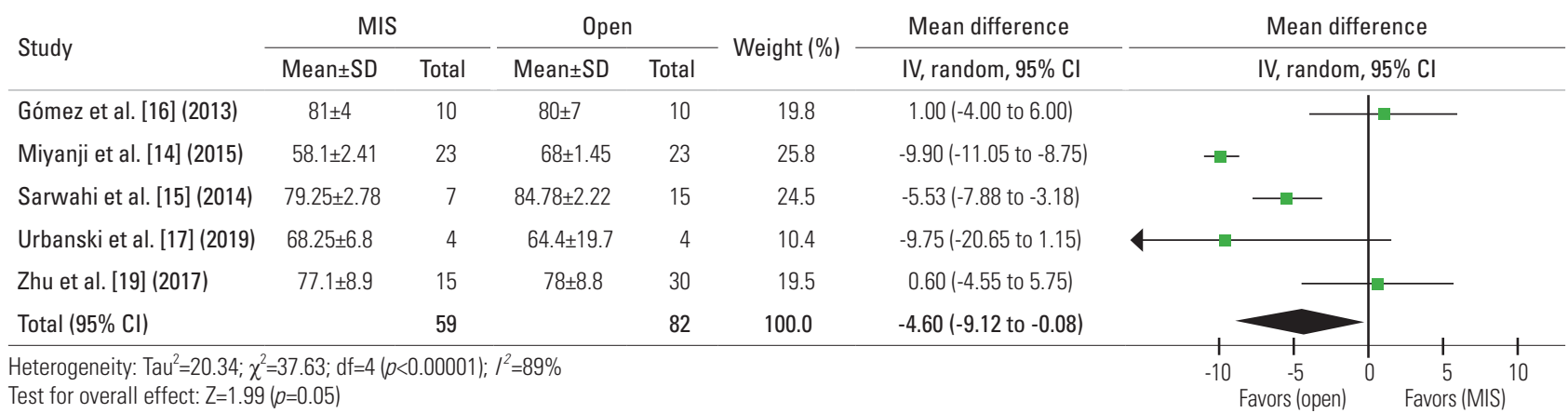

(C)

\begin{tabular}{|c|c|c|c|c|c|c|c|c|c|c|}
\hline \multirow{3}{*}{$\begin{array}{l}\text { Study } \\
\text { Miyanji et al. [14] (2015) }\end{array}$} & \multicolumn{2}{|c|}{ MIS } & \multicolumn{2}{|c|}{ Open } & \multirow{3}{*}{$\begin{array}{c}\text { Weight (\%) } \\
86.7\end{array}$} & \multirow{3}{*}{$\begin{array}{c}\text { Mean difference } \\
\text { IV, random, } 95 \% \mathrm{Cl} \\
-0.11(-0.18 \text { to }-0.04)\end{array}$} & \multirow{2}{*}{\multicolumn{3}{|c|}{$\begin{array}{c}\text { Mean difference } \\
\mathrm{IV} \text {, random, } 95 \% \mathrm{Cl}\end{array}$}} & \\
\hline & \multirow{2}{*}{$\begin{array}{c}\text { Mean } \pm \text { SD } \\
4.23 \pm 0.14\end{array}$} & \multirow{2}{*}{$\begin{array}{c}\text { Total } \\
23\end{array}$} & \multirow{2}{*}{$\begin{array}{c}\text { Mean } \pm \text { SD } \\
4.34 \pm 0.1\end{array}$} & \multirow{2}{*}{$\begin{array}{r}\text { Total } \\
23\end{array}$} & & & & & & \\
\hline & & & & & & & & - & & \\
\hline Si et al. [18] (2020) & $4 \pm 0.4$ & 64 & $4.2 \pm 0.7$ & 48 & 8.8 & $-0.20(-0.42$ to 0.02$)$ & & & & \\
\hline Zhu et al. [19] (2017) & $4.3 \pm 0.5$ & 15 & $4.2 \pm 0.5$ & 30 & 4.5 & $0.10(-0.21$ to 0.41$)$ & & & 一ـ & \\
\hline Total $(95 \% \mathrm{Cl})$ & & 102 & & 101 & 100.0 & $-0.11(-0.17$ to -0.04$)$ & & & & \\
\hline $\begin{array}{l}\text { Heterogeneity: } \chi^{2}=2.40 ; d \\
\text { Test for overall effect: } Z=3\end{array}$ & $\begin{array}{l}(p=0.30) ; /^{2} \\
(p=0.001)\end{array}$ & & & & & & -0.5 & $\begin{array}{c}-0.25 \\
\text { Favors (open) }\end{array}$ & $\begin{array}{cc}0 & 0.25 \\
& \text { Favors (MIS) }\end{array}$ & 0.5 \\
\hline
\end{tabular}

Fig. 2. Showing forest plots of primary outcomes. (A) Forest plot of correction rate at final follow-up. (B) Forest plot showing sensitivity analysis of correction rate at final follow-up. (C) Forest plot of functional outcome using Scoliosis Research Society-22 (SRS-22) score at final follow-up. MIS, minimally invasive surgery; SD, standard deviation; IV, inverse variance; $\mathrm{Cl}$, confidence interval; df, degrees of freedom.

\section{Blood loss}

All six included studies provided data regarding intraoperative blood loss. The quantitative analysis results favored the MIS group ( $p<0.00001$; mean difference, $279.21 ; 95 \%$ CI, 211.76 to 346.67) (Fig. 3B). This can be attributed to more exposure in the open surgery group and smaller incisions in the MIS group. Blood loss is a significant parameter; it makes the patient unstable and requires transfusion.

\section{Number of patients requiring transfusion}

Only three out of six studies mentioned the number of patients requiring transfusion in each group. There were 75 patients in the MIS group and 67 patients in the open surgery group. We used the Mantel-Haenszel method for the quantitative analysis using the fixed-effects model. The results favored the MIS group shown in Fig. 3C ( $p<0.0001$; OR, 0.20 ; $95 \% \mathrm{CI}, 0.10$ to 0.42 ). This shows low transfusion rates in the MIS group. 
(A)

\begin{tabular}{|c|c|c|c|c|c|c|c|c|}
\hline \multirow{3}{*}{$\begin{array}{l}\text { Study } \\
\text { Gómez et al. [16] (2013) }\end{array}$} & \multicolumn{2}{|l|}{ MIS } & \multicolumn{2}{|l|}{ Open } & \multirow{3}{*}{$\begin{array}{c}\text { Weight (\%) } \\
5.8\end{array}$} & \multirow{3}{*}{$\begin{array}{c}\text { Mean difference } \\
\text { IV, random, } 95 \% \mathrm{Cl} \\
162.00 \text { (38.21 to } 285.79 \text { ) }\end{array}$} & \multirow{2}{*}{\multicolumn{2}{|c|}{$\begin{array}{c}\text { Mean difference } \\
\text { IV, random, } 95 \% \mathrm{CI}\end{array}$}} \\
\hline & \multirow{2}{*}{$\begin{array}{r}\text { Mean } \pm \text { SD } \\
366 \pm 168\end{array}$} & \multirow{2}{*}{$\begin{array}{r}\text { Total } \\
10\end{array}$} & \multirow{2}{*}{$\begin{array}{c}\text { Mean } \pm \text { SD } \\
204 \pm 108\end{array}$} & \multirow{2}{*}{$\begin{array}{r}\text { Total } \\
10\end{array}$} & & & & \\
\hline & & & & & & & & $\longrightarrow$ \\
\hline Miyanji et al. [14] (2015) & $475.3 \pm 13.25$ & 23 & $346.4 \pm 15.64$ & 23 & 27.4 & 128.90 (120.52 to 137.28$)$ & & - \\
\hline Sarwahi et al. [15] (2014) & $538.8 \pm 22.2$ & 15 & $424.2 \pm 144$ & 15 & 11.7 & 114.60 (39.89 to 189.31 ) & & \\
\hline Si et al. [18] (2020) & $361 \pm 95$ & 64 & $275 \pm 43$ & 48 & 23.8 & 86.00 (59.74 to 112.26$)$ & & - \\
\hline Urbanski et al. [17] (2019) & $285 \pm 47.56$ & 4 & $242 \pm 44.51$ & 4 & 13.9 & 43.00 (-20.84 to 106.84$)$ & & \\
\hline Zhu et al. [19] (2017) & $252 \pm 96$ & 15 & $192 \pm 30$ & 30 & 17.3 & 60.00 (10.25 to 109.75) & & - - \\
\hline Total $(95 \% \mathrm{CI})$ & & 123 & & 130 & 100.0 & 95.06 (61.43 to 128.70$)$ & & \\
\hline eterogeneity: lau $=1,056.94$ & $00001)$ & & & & & & $-200-100$ & $\begin{array}{c}100 \quad 20 \\
\text { Favors (open) }\end{array}$ \\
\hline
\end{tabular}

(B)

\begin{tabular}{|c|c|c|c|c|c|c|c|c|c|}
\hline \multirow{3}{*}{$\begin{array}{l}\text { Study } \\
\text { Gómez et al. [16] (2013) }\end{array}$} & \multicolumn{2}{|l|}{ MIS } & \multicolumn{2}{|l|}{ Open } & \multirow{2}{*}{$\begin{array}{c}\text { Weight } \\
(\%)\end{array}$} & \multirow{2}{*}{$\begin{array}{l}\text { Mean difference } \\
\mathrm{IV} \text {, random, } 95 \% \mathrm{Cl}\end{array}$} & \multirow{2}{*}{\multicolumn{3}{|c|}{$\begin{array}{c}\text { Mean difference } \\
\mathrm{IV} \text {, random, } 95 \% \mathrm{Cl}\end{array}$}} \\
\hline & \multirow{2}{*}{$\begin{array}{c}\text { Mean } \pm \text { SD } \\
270 \pm 95\end{array}$} & \multirow{2}{*}{$\begin{array}{c}\text { Total } \\
10\end{array}$} & \multirow{2}{*}{$\begin{array}{r}\text { Mean } \pm \text { SD } \\
720 \pm 177.5\end{array}$} & \multirow{2}{*}{$\begin{array}{c}\text { Total } \\
10\end{array}$} & & & & & \\
\hline & & & & & 13.9 & $-450.00(-574.78$ to -325.22$)$ & $1=$ & & \\
\hline Miyanji et al. [14] (2015) & $261.5 \pm 20.89$ & 23 & $471.7 \pm 36.09$ & 23 & 26.0 & $-210.20(-227.24$ to -193.16$)$ & - & & \\
\hline Sarwahi et al. [15] (2014) & $600 \pm 137.5$ & 7 & $800 \pm 162.5$ & 15 & 13.2 & $-200.00(-330.91$ to -69.09$)$ & - & & \\
\hline Si et al. [18] (2020) & $502 \pm 218$ & 64 & $808 \pm 520$ & 48 & 10.9 & $-306.00(-462.50$ to -149.50$)$ & & & \\
\hline Urbanski et al. [17] (2019) & $138.75 \pm 50.04$ & 4 & $450 \pm 106.06$ & 4 & 15.0 & $-311.25(-426.17$ to -196.33$)$ & - & & \\
\hline Zhu et al. [19] (2017) & $153 \pm 97$ & 15 & $418 \pm 126$ & 30 & 21.0 & $-265.00(-331.65$ to -198.35$)$ & - & & \\
\hline Total $(95 \% \mathrm{CI})$ & & 123 & & 130 & 100.0 & $-279.21(-346.67$ to -211.76$)$ & & & \\
\hline $\begin{array}{l}\text { Heterogeneity: } \text { Tau }^{2}=4,481.29 \\
\text { Test for overall effect: } Z=8.11\end{array}$ & $\begin{array}{l}=19.74 ; \mathrm{df}= \\
0.00001)\end{array}$ & & ${ }^{2}=75 \%$ & & & & $\begin{array}{l}-250 \\
\text { Favors (MIS) }\end{array}$ & $\begin{array}{rr}1 & 250 \\
0 & \text { Favors (open) }\end{array}$ & 500 \\
\hline
\end{tabular}

(C)

\begin{tabular}{|c|c|c|c|c|c|c|c|c|c|c|}
\hline \multirow{3}{*}{$\begin{array}{l}\text { Study } \\
\text { Sarwahi et al. [15] (2014) }\end{array}$} & \multicolumn{2}{|c|}{ MIS } & \multicolumn{2}{|c|}{ Open } & \multirow{2}{*}{$\begin{array}{l}\text { Weight } \\
(\%)\end{array}$} & \multirow{2}{*}{$\frac{\mathrm{OR}}{\mathrm{M}-\mathrm{H}, \text { fixed, } 95 \% \mathrm{Cl}}$} & \multicolumn{4}{|c|}{$\mathrm{OR}$} \\
\hline & Events & Total & Events & Total & & & \multicolumn{4}{|c|}{$\mathrm{M}-\mathrm{H}$, fixed, $95 \% \mathrm{Cl}$} \\
\hline & 1 & 7 & 11 & 15 & 18.4 & 0.06 (0.01 to 0.67$)$ & & $\longrightarrow$ & - & \\
\hline Si et al. [18] (2020) & 17 & 64 & 28 & 48 & 72.0 & 0.26 (0.12 to 0.57$)$ & & & & \\
\hline Zhu et al. [19] (2017) & 0 & 4 & 3 & 4 & 9.6 & 0.05 (0.00 to 1.56$)$ & & . & - & \\
\hline Total $(95 \% \mathrm{Cl})$ & & 75 & & 67 & 100.0 & $0.20(0.10$ to 0.42$)$ & & & & \\
\hline Total events & 18 & & 42 & & & & & & & \\
\hline $\begin{array}{l}\text { Heterogeneity: } \chi^{2}=1.99 ; d f=2 \\
\text { Test for overall effect: } Z=4.33\end{array}$ & $=0.37) ; /$ & & & & & & 0.001 & $\begin{array}{c}0.1 \\
\text { Favors (MIS) }\end{array}$ & $\begin{array}{lc}1 & 10 \\
& \text { Favors (open) }\end{array}$ & 1,000 \\
\hline
\end{tabular}

(D)

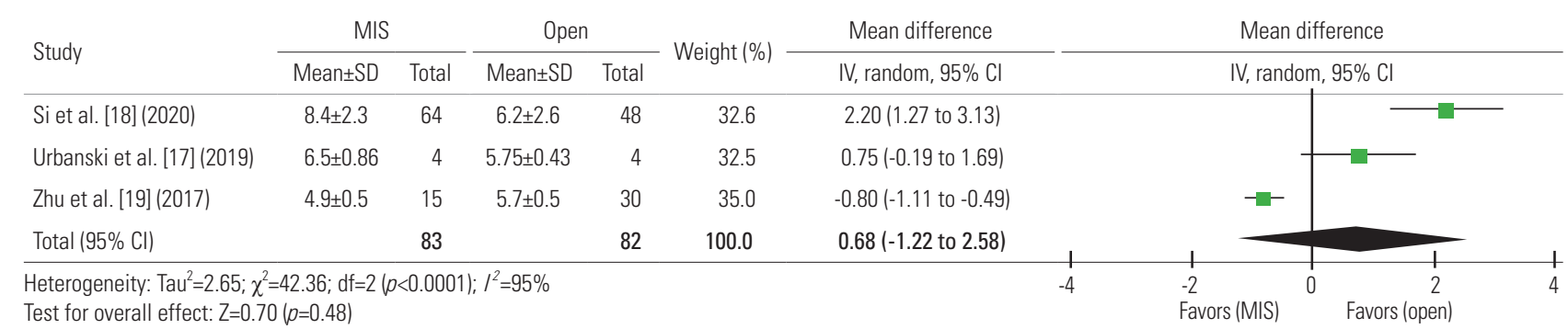

Fig. 3. (A) Forest plot of duration of surgery. (B) Forest plot of intra-operative blood loss. (C) Forest plot of transfusion requirement. (D) Forest plot of number of levels fused. MIS, minimally invasive surgery; SD, standard deviation; IV, inverse variance; Cl, confidence interval; df, degrees of freedom; OR, odds ratio; M-H, MantelHaenszel test. 


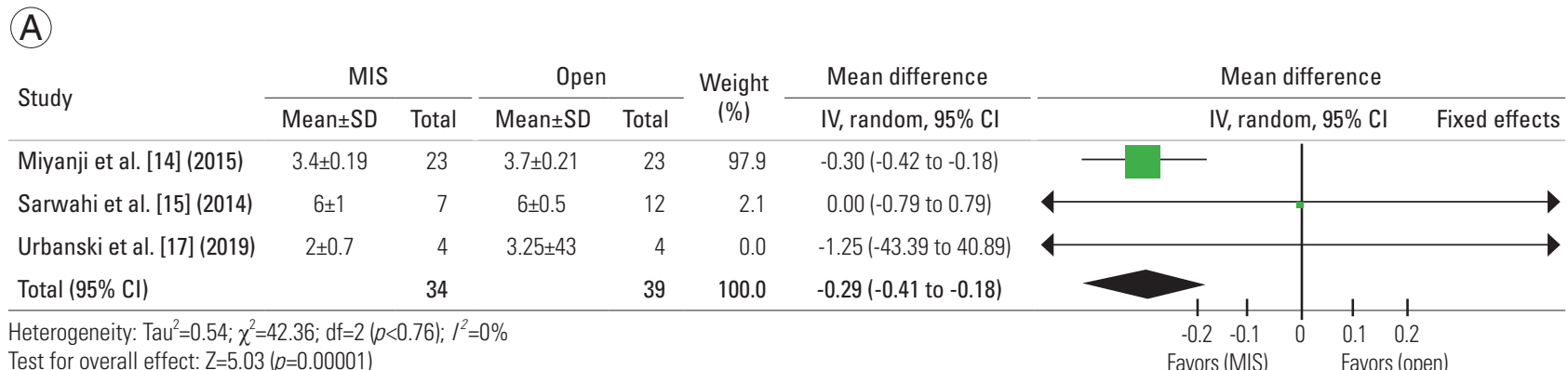

(B)

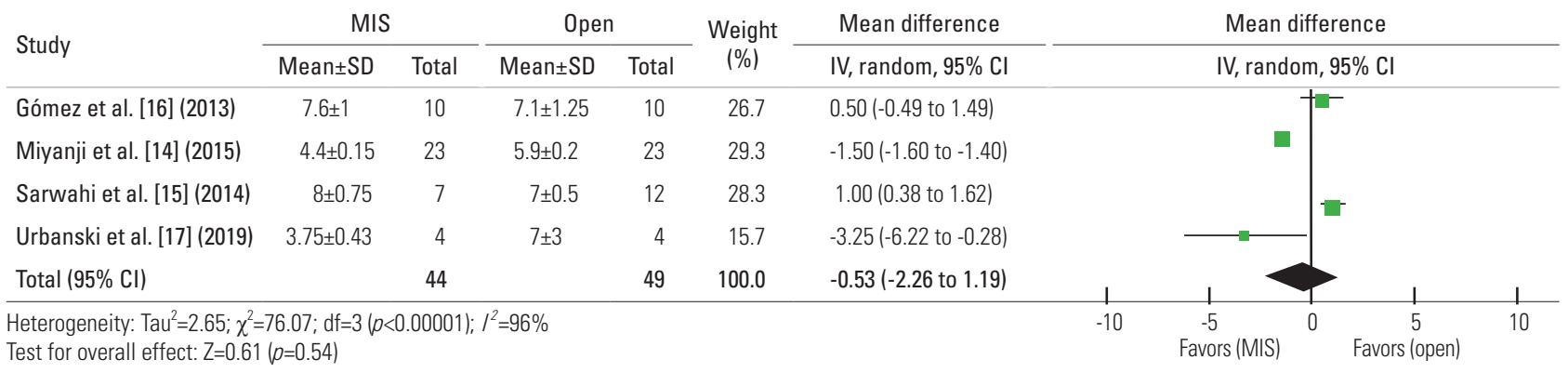

(C)

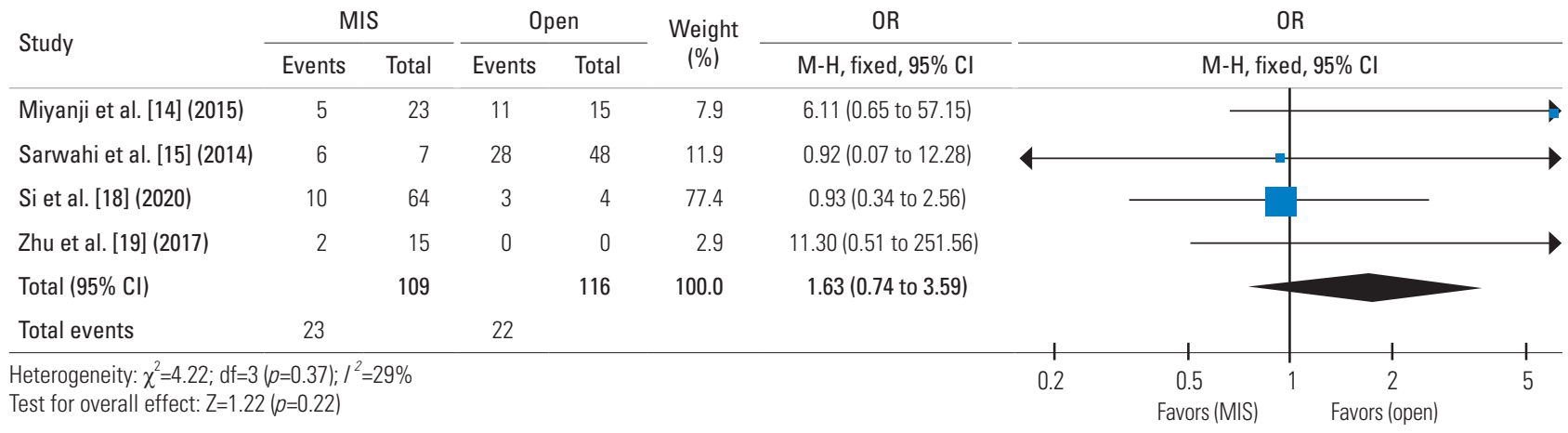

Fig. 4. (A) Forest plot of number of days requiring analgesia. (B) Forest plot of length of hospital stay. (C) Forest plot of complications. PCA, patient-controlled analgesia; MIS, minimally invasive surgery; SD, standard deviation; IV, inverse variance; Cl, confidence interval; df, degrees of freedom; OR, odds ratio; M-H, Mantel-Haenszel test.

\section{Number of levels fused}

Three out of six included studies mentioned data of the number of levels fused in each group. There were 83 patients in the MIS group and 82 patients in the open group. We used a random-effects model given high heterogeneity. The results showed no difference between the two groups ( $p=0.48$; 95\% CI, 1.22 to 2.58 ) (Fig. 3D). This indicates that even using the MIS technique; we can achieve the same number of fusion levels.

\section{Patient-controlled analgesia or opioid requirement}

Three out of six studies provided data regarding patient- controlled analgesia (PCA) or opioid requirements in each group. There were 34 patients in the MIS group and 39 patients in the open surgery group. The quantitative analysis favored the MIS group $(p<0.00001$; mean difference, $0.29 ; 95 \%$ CI, 0.18 to 0.41 ). We used a fixed-effects model considering low heterogeneity. MIS was probably favored here because of a lower occurrence of tissue damage than in open surgery (Fig. 4A).

\section{Length of stay}

Only four out of six studies mentioned the postoperative length of stay (44 patients in the MIS group and 49 patients in the open group). The quantitative analysis 
showed no significant difference between the two groups ( $p=0.54 ; 95 \%$ CI, 1.19 to 2.26 ). We used a random-effects model for this analysis, given high heterogeneity. While the meta-analysis slightly favored the MIS group, the difference was not significant; this could be due to the small sample sizes in each study (Fig. 4B).

\section{Complications}

Pseudoarthrosis, implant failure, respiratory complications, neural injury, additional surgery, and infection were considered postoperative complications. Four out of six studies mentioned data on complications in the two groups. There were 109 patients in the MIS group and 116 in the open surgery group. We used the Mantel-Haenszel model for this analysis, and the fixed-effects model was applied as the heterogeneity was low. The quantitative analysis favored the open surgery group, but the results were not significant. This could be because of the low sample size. Implant-related complications, like hardware failure, were higher in the MIS ( $n=5)$ group than the open surgery ( $\mathrm{n}=2)$ group (Fig. 4 C).

\section{Discussion}

AIS is a complex 3D deformity mainly affecting girls between the ages of 10-18 years. While most patients can be managed conservatively, surgery might be required in symptomatic and severely deformed patients. People also opt for surgery for cosmetic reasons. The surgeon usually uses a posterior approach to expose all the levels of the deformed vertebra. This approach is considered a gold standard for AIS patients because the surgeon can address all the deformities and perform facetectomies and fusions adequately. MIS has recently become popular for many orthopedic conditions; it has also been applied to the spine to perform discectomies, fusions, and pedicle screw fixations. Mundis et al. [20] showed a significant improvement of outcome with MIS in adult deformity corrections. However, we have minimal data on MIS for surgically correcting AIS. De Bodman et al. [21] showed a significant improvement in deformity correction using the MIS technique. They also found low estimated blood loss and shorter stay using this technique. We only found a few studies comparing the two techniques, MIS and open surgery.

The primary outcome of deformity correction surgery is adequate deformity correction and improved functional outcomes. Gómez et al. [16] and Si et al. [18] showed a better correction rate in the MIS group, but the difference was not significant in their comparative studies. Other studies demonstrated better correction rates in the open surgery group. Our meta-analysis found that the results favored the open surgery group, but the difference was not statistically significant. Sensitivity analysis showed statistically significant results favoring open surgery for achieving a good correction rate at the final follow-up. Also, studies mention better functional outcomes using SRS-22 scores with the open surgery technique.

The secondary outcome parameters included intraoperative blood loss, surgery duration, transfusion requirement, length of stay, analgesic requirement, and complications. Even though open surgery involves extensive dissection, it takes less time to complete. This could be because, after adequate exposure, it is easier for a surgeon to perform all steps conveniently under direct vision. The MIS technique took longer, possibly because it would be challenging to do facetectomies, fusions, and screw placings in the rotated vertebrae with limited vision. Considering the amount of soft tissue dissected, anticipated blood loss will be higher in the open surgery technique. Our analysis favored the MIS group with respect to blood loss. These results are also consistent with another analysis where fewer patients required blood transfusion in the MIS group. More patients required blood transfusion in the open surgery group. Although there are studies showing tranexamic acid used to decrease blood loss [22,23], MIS had a clear advantage over the open surgery group pertaining to this parameter. A meta-analysis also showed no difference between the two groups in the number of levels fused. This suggests that MIS is as good as the open surgery technique in attaining multilevel vertebral fusions. Urbanski et al. [17] showed a significant difference in the number of days opioids were required, with a shorter period in the MIS group. Sarwahi et al. [15] showed no difference between the two groups in opioid requirements, and our analysis favored the MIS group in this parameter. Gómez et al. [16] and Sarwahi et al. [15] showed a short duration of stay in the open surgery group, whereas Miyanji and Desai [14] and Urbanski et al. [17] showed a shorter stay in the MIS group. Our analysis showed no difference between both groups in this parameter. Miyanji and Desai [14], Si et al. [18], and Zhu et al. [19] reported a higher number of complications in the MIS group; our 
analysis favored the open surgery group in this parameter, the difference was not statistically significant. The difference could be because not all studies reported complications. Three studies mentioned the accuracy of screw placement in both groups. Sarwahi et al. [15] reported an accuracy rate of $90.7 \%$ using the freehand technique in the MIS group, Zhu et al. [19] reported an accuracy of 93.8\% using O-arm navigation in the MIS group, and Si et al. [18] reported an accuracy rate of $94.1 \%$ in MIS group using a freehand technique. This meta-analysis showed that the traditional posterior approach had a clear advantage over MIS in achieving better correction rates and functional outcomes. Our analysis also showed MIS's advantages over open surgery as a shorter surgery duration, decreased blood loss, lower transfusion requirements, and analgesia (PCA or opioid) requirement. However, again, there arises a dilemma regarding which parameters are more critical. Considering a deformity correction surgery, a surgical technique should provide a better correction rate than the other. Open surgery has a clear advantage of achieving a better correction rate and good functional outcome scores. This analysis has limitations, like none of the studies were RCTs, and most of them were retrospective in nature with a small sample size. Although there was no significant difference among the two groups when comparing coronal and sagittal balance, the values were not similar in each study. The type of curve was also not similar in each study. This identifies the need for future studies with prospective RCT to suggest that one method has superiority over the other through level 1 evidence.

\section{Conclusions}

Open surgery is better than MIS surgery for AIS patients to achieve correction and good functional outcomes. Although MIS has benefits such as shorter surgery duration and blood loss, its role in AIS should be studied further in AIS patients.

\section{Conflict of Interest}

No potential conflict of interest relevant to this article was reported.

\section{References}

1. Kindsfater K, Lowe T, Lawellin D, Weinstein D, Ak- makjian J. Levels of platelet calmodulin for the prediction of progression and severity of adolescent idiopathic scoliosis. J Bone Joint Surg Am 1994;76:118692.

2. Nowak R, Kwiecien M, Tkacz M, Mazurek U. Transforming growth factor-beta (TGF- $\beta$ ) signaling in paravertebral muscles in juvenile and adolescent diopathic scoliosis. Biomed Res Int 2014;2014:594287.

3. Leboeuf D, Letellier K, Alos N, Edery P, Moldovan F. Do estrogens impact adolescent idiopathic scoliosis? Trends Endocrinol Metab 2009;20:147-52.

4. Justice CM, Miller NH, Marosy B, Zhang J, Wilson AF. Familial idiopathic scoliosis: evidence of an Xlinked susceptibility locus. Spine (Phila Pa 1976) 2003;28:589-94.

5. Stokes IA, Spence H, Aronsson DD, Kilmer N. Mechanical modulation of vertebral body growth. Implications for scoliosis progression. Spine (Phila $\mathrm{Pa}$ 1976) 1996;21:1162-7.

6. Kamel I, Barnette R. Positioning patients for spine surgery: avoiding uncommon position-related complications. World J Orthop 2014;5:425-43.

7. Carreon LY, Puno RM, Lenke LG, et al. Non-neurologic complications following surgery for adolescent idiopathic scoliosis. J Bone Joint Surg Am 2007;89:2427-32.

8. Coe JD, Arlet V, Donaldson W, et al. Complications in spinal fusion for adolescent idiopathic scoliosis in the new millennium: a report of the Scoliosis Research Society Morbidity and Mortality Committee. Spine (Phila Pa 1976) 2006;31:345-9.

9. McAfee PC, Phillips FM, Andersson G, et al. Minimally invasive spine surgery. Spine (Phila Pa 1976) 2010;35(26 Suppl):S271-3.

10. Yang JH, Chang DG, Suh SW, et al. Safety and effectiveness of minimally invasive scoliosis surgery for adolescent idiopathic scoliosis: a retrospective case series of 84 patients. Eur Spine J 2020;29:761-9.

11. Tarpada SP, Morris MT. Minimally invasive surgery in the treatment of adolescent idiopathic scoliosis: a literature review and meta-analysis. J Orthop 2016;14:19-22.

12. Moher D, Liberati A, Tetzlaff J, Altman DG; PRISMA Group. Preferred Reporting Items for Systematic Reviews and Meta-Analyses: the PRISMA statement. PLoS Med 2009;6:e1000097.

13. Slim K, Nini E, Forestier D, Kwiatkowski F, Panis Y, 
Chipponi J. Methodological index for non-randomized studies (minors): development and validation of a new instrument. ANZ J Surg 2003;73:712-6.

14. Miyanji F, Desai S. Minimally invasive surgical options for adolescent idiopathic scoliosis. Semin Spine Surg 2015;27:39-44.

15. Sarwahi V, Horn JJ, Kulkarni PM, et al. Minimally invasive surgery in patients with adolescent idiopathic scoliosis: is it better than the standard approach?: a 2-year follow-up study. Clin Spine Surg 2016;29:33140.

16. Gomez H, Burgos J, Hevia E, Maruenda JI, Barrios C, Sanpera I. Immediate postoperative and long-term results of a minimally invasive approach for the correction of adolescent idiopathic scoliosis. Coluna/ Columna 2013;12:291-5.

17. Urbanski W, Zaluski R, Kokaveshi A, Aldobasic S, Miekisiak G, Morasiewicz P. Minimal invasive posterior correction of Lenke 5C idiopathic scoliosis: comparative analysis of minimal invasive vs. open surgery. Arch Orthop Trauma Surg 2019;139:1203-8.

18. Si G, Li, T, Wang Y, Liu X, Li C, Yu M. Minimally invasive surgery versus standard posterior approach for Lenke type 1-4 adolescent idiopathic scoliosis: a multicenter, retrospective study. Eur Spine J 2020;30:70613.
19. Zhu W, Sun W, Xu L, et al. Minimally invasive scoliosis surgery assisted by $\mathrm{O}$-arm navigation for Lenke type $5 \mathrm{C}$ adolescent idiopathic scoliosis: a comparison with standard open approach spinal instrumentation. J Neurosurg Pediatr 2017;19:472-8.

20. Mundis GM, Akbarnia BA, Phillips FM. Adult deformity correction through minimally invasive lateral approach techniques. Spine (Phila Pa 1976) 2010;35(26 Suppl):S312-21.

21. De Bodman C, Miyanji F, Borner B, Zambelli PY, Racloz G, Dayer R. Minimally invasive surgery for adolescent idiopathic scoliosis: correction of deformity and peri-operative morbidity in 70 consecutive patients. Bone Joint J 2017;99-B:1651-7.

22. Choi HY, Hyun SJ, Kim KJ, Jahng TA, Kim HJ. Effectiveness and safety of tranexamic acid in spinal deformity surgery. J Korean Neurosurg Soc 2017;60:7581.

23. Alajmi T, Saeed H, Alfaryan K, Alakeel A, Alfaryan T. Efficacy of tranexamic acid in reducing blood loss and blood transfusion in idiopathic scoliosis: a systematic review and meta-analysis. J Spine Surg 2017;3:531-40. 